\title{
Gilbert Damping in Noncollinear Ferromagnets
}

\author{
Zhe Yuan, ${ }^{1, *}$ Kjetil M. D. Hals, ${ }^{2,3}$ Yi Liu, ${ }^{1}$ Anton A. Starikov, ${ }^{1}$ Arne Brataas, ${ }^{2}$ and Paul J. Kelly ${ }^{1}$ \\ ${ }^{1}$ Faculty of Science and Technology and MESA ${ }^{+}$Institute for Nanotechnology, University of Twente, \\ P.O. Box 217, 7500 AE Enschede, The Netherlands \\ ${ }^{2}$ Department of Physics, Norwegian University of Science and Technology, NO-7491 Trondheim, Norway \\ ${ }^{3}$ Niels Bohr International Academy and the Center for Quantum Devices, Niels Bohr Institute, \\ University of Copenhagen, 2100 Copenhagen, Denmark \\ (Received 4 April 2014; revised manuscript received 20 November 2014; published 31 December 2014)
}

\begin{abstract}
The precession and damping of a collinear magnetization displaced from its equilibrium are well described by the Landau-Lifshitz-Gilbert equation. The theoretical and experimental complexity of noncollinear magnetizations is such that it is not known how the damping is modified by the noncollinearity. We use first-principles scattering theory to investigate transverse domain walls (DWs) of the important ferromagnetic alloy $\mathrm{Ni}_{80} \mathrm{Fe}_{20}$ and show that the damping depends not only on the magnetization texture but also on the specific dynamic modes of Bloch and Néel DWs in ways that were not theoretically predicted. Even in the highly disordered $\mathrm{Ni}_{80} \mathrm{Fe}_{20}$ alloy, the damping is found to be remarkably nonlocal.
\end{abstract}

DOI: 10.1103/PhysRevLett.113.266603

PACS numbers: 72.25.Rb, 75.60.Ch, 75.60.Jk, 75.78.-n

Introduction.-The key common ingredient in various proposed nanoscale spintronics devices involving magnetic droplet solitons [1], Skyrmions [2,3], or magnetic domain walls (DWs) [4,5], is a noncollinear magnetization that can be manipulated using current-induced torques (CITs) [6]. Different microscopic mechanisms have been proposed for the CIT including spin transfer [7,8], spin-orbit interaction with broken inversion symmetry in the bulk or at interfaces [9-11], the spin-Hall effect [12], or proximity-induced anisotropic magnetic properties in adjacent normal metals [13]. Their contributions are hotly debated but can only be disentangled if the Gilbert damping torque is accurately known. This is not the case [14]. Theoretical work [15-19] suggesting that noncollinearity can modify the Gilbert damping due to the absorption of the pumped spin current by the adjacent precessing magnetization has stimulated experimental efforts to confirm this quantitatively $[14,20]$. In this Letter, we use first-principles scattering calculations to show that the Gilbert damping in a noncollinear alloy can be significantly enhanced depending on the particular precession modes and surprisingly, that even in a highly disordered alloy like $\mathrm{Ni}_{80} \mathrm{Fe}_{20}$, the nonlocal character of the damping is very substantial. Our findings are important for understanding field- and/or current-driven noncollinear magnetization dynamics and for designing new spintronics devices.

Gilbert damping in $\mathrm{Ni}_{80} \mathrm{Fe}_{20}$ DWs.-Gilbert damping is in general described by a symmetric $3 \times 3$ tensor. For a substitutional, cubic binary alloy like Permalloy, $\mathrm{Ni}_{80} \mathrm{Fe}_{20}$, this tensor is essentially diagonal and isotropic and reduces to scalar form when the magnetization is collinear. A value of this dimensionless scalar calculated from first principles, $\alpha_{\text {coll }}=0.0046$, is in good agreement with values extracted from room-temperature experiments that range between 0.004 and 0.009 [21]. In a one-dimensional (1D) transverse DW, the Gilbert damping tensor is still diagonal but, as a consequence of the lowered symmetry [22], it contains two unequal components. The magnetization in static Néel or Bloch DWs lies inside well-defined planes that are illustrated in Fig. 1. An angle $\theta$ represents the in-plane rotation with respect to the magnetization in the left domain and it varies from 0 to $\pi$ through a $180^{\circ} \mathrm{DW}$. If the plane changes in time, as it does when the magnetization precesses, an angle $\phi$ can be used to describe its rotation. We define an out-of-plane damping component $\alpha_{\mathrm{o}}$ corresponding to variation in $\phi$, and an in-plane component $\alpha_{\mathrm{i}}$ corresponding to time-dependent $\theta$. Rigid translation of the DW, i.e., making the DW center $r_{w}$ vary in time, is a specific example of the latter.

For Walker-profile DWs [23], an effective (dimensionless) in-plane $\left(\alpha_{i}^{\text {eff }}\right)$ and out-of-plane damping $\left(\alpha_{\mathrm{o}}^{\text {eff }}\right)$ can be calculated in terms of the scattering matrix $S$ of the system using the scattering theory of magnetization dissipation $[24,25]$. Both calculated values are plotted in Fig. 1(c) as a function of the inverse DW width $1 / \lambda_{w}$ for Néel and Bloch DWs. Results with the spin-orbit coupling (SOC) artificially switched off are shown for comparison; because spin space is then decoupled from real space, the results for the two DW profiles are identical and both $\alpha_{i}^{\text {eff }}$ and $\alpha_{0}^{\text {eff }}$ vanish in the large $\lambda_{w}$ limit confirming that SOC is the origin of intrinsic Gilbert damping for collinear magnetization. With SOC switched on, Néel and Bloch DWs have identical values within the numerical accuracy, reflecting the negligibly small magnetocrystalline anisotropy in Permalloy. Both $\alpha_{i}^{\text {eff }}$ and $\alpha_{0}^{\text {eff }}$ approach the collinear value $\alpha_{\text {coll }}$ [21], shown as a green dot in the figure, in the wide 
(a)

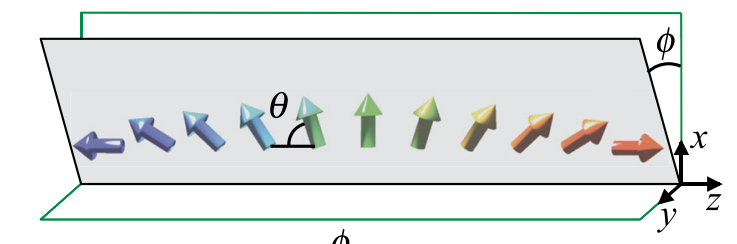

(b)
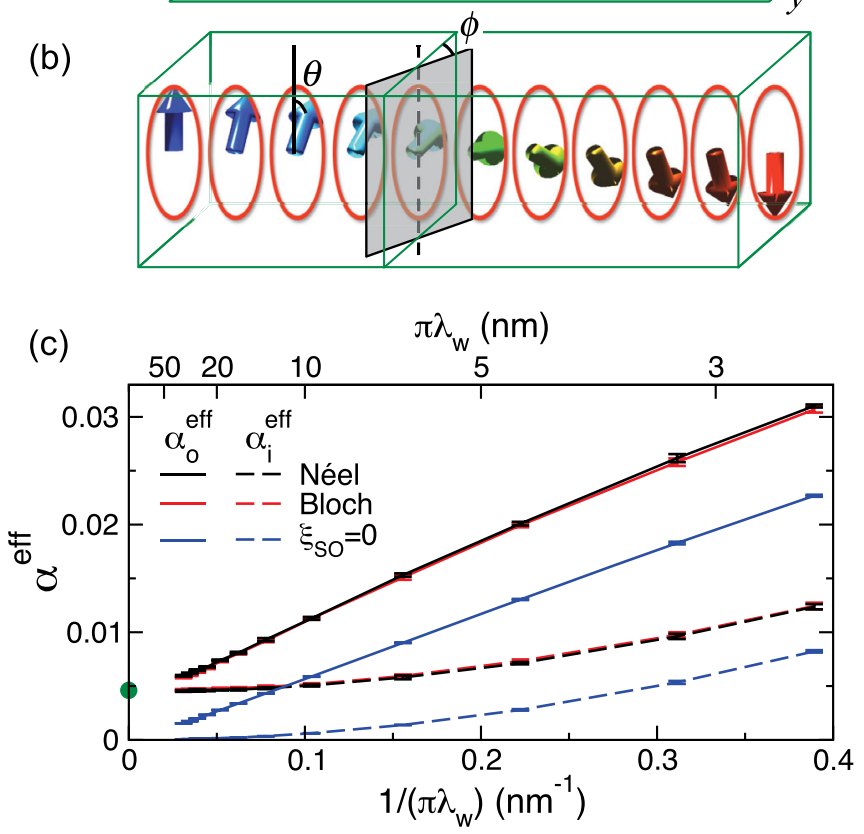

FIG. 1 (color online). Sketch of Néel (a) and Bloch (b) DWs. (c) Calculated effective Gilbert damping parameters for Permalloy DWs (Néel, black lines; Bloch, red lines) as a function of the inverse of the DW width $\lambda_{w}$. Without spin-orbit coupling, calculations for the two DW types yield the same results (blue lines). The green dot represents the value of Gilbert damping calculated for collinear Permalloy. For each value of $\lambda_{w}$, we typically consider eight different disorder configurations and the error bars are a measure of the spread of the results.

DW limit. For finite widths, they exhibit a quadratic and a predominantly linear dependence on $1 /\left(\pi \lambda_{w}\right)$, respectively, both with and without SOC; for large values of $\lambda_{w}$, there is a hint of nonlinearity in $\alpha_{0}^{\text {eff }}\left(1 / \lambda_{w}\right)$. However, phenomenological theories [15-17] predict that $\alpha_{\mathrm{i}}^{\text {eff }}$ should be independent of $\lambda_{w}$ and equal to $\alpha_{\text {coll }}$ while $\alpha_{0}^{\text {eff }}$ should be a quadratic function of the magnetization gradient. Neither of these predicted behaviors is observed in Fig. 1(c), indicating that existing theoretical models of texture-enhanced Gilbert damping need to be reexamined.

The $\alpha^{\text {eff }}$ shown in Fig. 1(c) is an effective damping constant because the magnetization gradient $d \theta / d z$ of a Walker-profile DW is inhomogeneous. Our aim in the following is to understand the physical mechanisms of texture-enhanced Gilbert damping with a view to determining how the local damping depends on the magnetization gradient, as well as the corresponding parameters for Permalloy, and finally to express these in a form suitable for use in micromagnetic simulations.

In-plane damping $\alpha_{\mathrm{i}}$.-To get a clearer picture of how the in-plane damping depends on the gradient, we calculate the
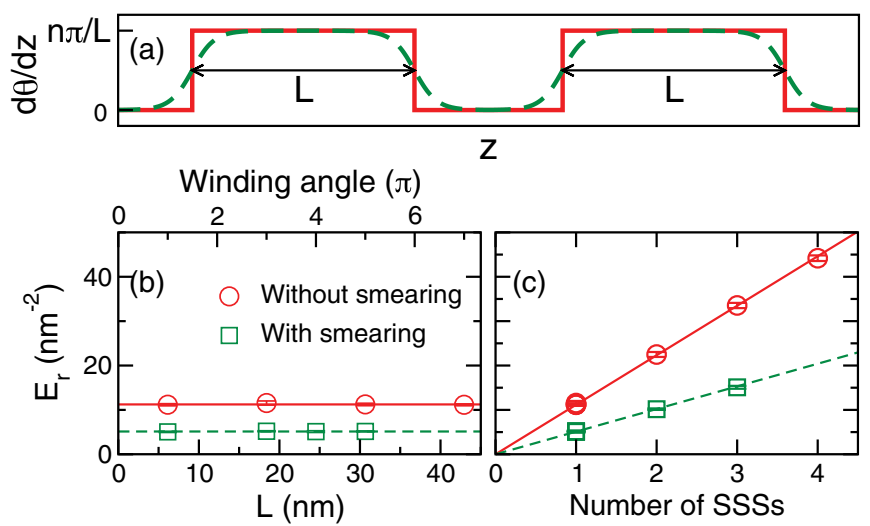

FIG. 2 (color online). (a) Sketch of the magnetization gradient for two SSSs separated by collinear magnetization with (green, dashed line) and without (red, solid line) a broadening of the magnetization gradient at the ends of the SSSs. The length of each segment is $L$. (b) Calculated energy pumping $E_{r}$ as a function of $L$ for a single Permalloy Bloch-DW-type SSS without SOC. The upper horizontal axis shows the total winding angle of the SSS. (c) Calculated energy pumping $E_{r}$ without SOC as a function of the number of SSSs that are separated by a stretch of collinear magnetization.

energy pumping $E_{r} \equiv \operatorname{Tr}\left[\left(\partial \boldsymbol{S} / \partial r_{s}\right)\left(\partial \boldsymbol{S}^{\dagger} / \partial r_{s}\right)\right]$ for a finite length $L$ of a Bloch-DW-type spin spiral (SS) centered at $r_{s}$. In this SS segment (SSS), $d \theta / d z$ is constant except at the ends. Figure 2(b) shows the results calculated without SOC for a single Permalloy SSS with $d \theta / d z=6^{\circ}$ per atomic layer; Fig. 1(c) showed that SOC does not influence the quadratic behavior essentially. $E_{r}$ is seen to be independent of $L$ indicating there is no dissipation when $d \theta / d z$ is constant in the absence of SOC. In this case, the only contribution arises from the ends of the SSS where $d \theta / d z$ changes abruptly; see Fig. 2(a). If we replace the step function of $d \theta / d z$ by a Fermi-like function with a smearing width equal to one atomic layer, $E_{r}$ decreases significantly (green squares). For multiple SSSs separated by collinear magnetization, we find that $E_{r}$ is proportional to the number of segments; see Fig. 2(c).

What remains is to understand the physical origin of the damping at the ends of the SSSs. Rigid translation of a SSS or of a DW allows for a dissipative spin current $j_{s}^{\prime \prime} \sim-\mathbf{m} \times \partial_{z} \partial_{t} \mathbf{m}$ that breaks time-reversal symmetry [19]. The divergence of $\boldsymbol{j}_{s}^{\prime \prime}$ gives rise to a local dissipative torque, whose transverse component is the enhancement of the in-plane Gilbert damping from the magnetization texture $\mathbf{m}(\mathbf{r})$. After straightforward algebra, we obtain the textureenhanced in-plane damping torque

$$
\alpha^{\prime \prime}\left[\left(\mathbf{m} \cdot \partial_{z} \partial_{t} \mathbf{m}\right) \mathbf{m} \times \partial_{z} \mathbf{m}-\mathbf{m} \times \partial_{z}^{2} \partial_{t} \mathbf{m}\right],
$$

where $\alpha^{\prime \prime}$ is a material parameter with dimensions of length squared. In 1D SSSs or DWs, Eq. (1) leads to the local energy dissipation rate $\dot{E}(\mathbf{r})=\left(\alpha^{\prime \prime} M_{s} / \gamma\right) \partial_{t} \theta \partial_{t}\left(d^{2} \theta / d z^{2}\right)$ [25], where $M_{s}$ is the saturation magnetization and $\gamma=$ $g \mu_{B} / \hbar$ is the gyromagnetic ratio expressed in terms of the 
Landé $g$ factor and the Bohr magneton $\mu_{B}$. This result shows explicitly that the in-plane damping enhancement is related to finite $d^{2} \theta / d z^{2}$. Using the calculated data in Fig. 1(c), we extract a value for the coefficient $\alpha^{\prime \prime}=$ $0.016 \mathrm{~nm}^{2}$ that is independent of specific textures $\mathbf{m}(\mathbf{r})$ [25].

Out-of-plane damping $\alpha_{\mathrm{o}}$. -We begin our analysis of the out-of-plane damping with a simple two-band free-electron DW model [25]. Because the linearity of the damping enhancement does not depend on SOC, we examine the SOC free case for which there is no difference between Néel and Bloch DW profiles and we use Néel DWs in the following. Without disorder, we can use the known $\phi$ dependence of the scattering matrix for this model [31] to obtain $\alpha_{0}^{\text {eff }}$ analytically,

$$
\begin{aligned}
\alpha_{\mathrm{o}}^{\mathrm{eff}} & =\frac{g \mu_{B}}{4 \pi A M_{s} \lambda_{w}} \sum_{\mathbf{k}_{\|}}\left(\left|r_{\uparrow \downarrow}^{\mathbf{k}_{\|}}\right|^{2}+\left|r_{\downarrow \uparrow}^{\mathbf{k}_{\|}}\right|^{2}+\left|t_{\uparrow \downarrow}^{\mathbf{k}_{\|}}\right|^{2}+\left|t_{\downarrow \uparrow}^{\mathbf{k}_{\|}}\right|^{2}\right) \\
& \approx \frac{g \mu_{B}}{4 \pi A M_{s} \lambda_{w}} \frac{h}{e^{2}} G_{\mathrm{Sh}},
\end{aligned}
$$

where $A$ is the cross sectional area and the convention used for the reflection $(r)$ and transmission $(t)$ probability amplitudes is shown in Fig. 3(a). Note that $\left|t_{\uparrow \downarrow}^{\mathbf{k}_{\|}}\right|^{2}$ and $\left|t_{\downarrow \uparrow}^{\mathbf{k}_{\|}}\right|^{2}$ are of the order of unity and much larger than the other two terms between the brackets unless the exchange splitting is very large and the DW width very small. It is then a good approximation to replace the quantities in brackets by the number of propagating modes at $\mathbf{k}_{\|}$to obtain the second line of Eq. (2), where $G_{\mathrm{Sh}}$ is the Sharvin conductance that only depends on the free-electron density. Equation (2) shows analytically that $\alpha_{\mathrm{o}}^{\text {eff }}$ is proportional to $1 / \lambda_{w}$ in the ballistic regime. This is reproduced by the results of numerical calculations for ideal free-electron DWs shown as black circles in Fig. 3(b).

Introducing site disorder [32] into the free-electron model results in a finite resistivity. The out-of-plane damping calculated for disordered free-electron DWs exhibits a transition as a function of its width. For narrow DWs (ballistic limit), $\alpha_{\mathrm{o}}^{\mathrm{eff}}$ is inversely proportional to $\lambda_{w}$ and the green, red, and blue circles in Fig. 3(b) tend to become parallel to the violet line for small values of $\lambda_{w}$. If $\lambda_{w}$ is sufficiently large, $\alpha_{\mathrm{o}}^{\text {eff }}$ becomes proportional to $\lambda_{w}^{-2}$ in agreement with phenomenological predictions [15-17] where the diffusive limit is assumed. This demonstrates the different behavior of $\alpha_{0}^{\text {eff }}$ in these two regimes.

We can construct an expression that describes both the ballistic and diffusive regimes by introducing an explicit spatial correlation in the nonlocal form of the out-of-plane Gilbert damping tensor that was derived using the fluctuation-dissipation theorem [15]

$$
\begin{aligned}
{\left[\alpha_{\mathrm{o}}\right]_{i j}\left(\mathbf{r}, \mathbf{r}^{\prime}\right) } & =\alpha_{\mathrm{coll}} \delta_{i j} \delta\left(\mathbf{r}-\mathbf{r}^{\prime}\right)+\alpha^{\prime} D\left(\mathbf{r}, \mathbf{r}^{\prime} ; l_{0}\right) \\
& \times\left[\mathbf{m}(\mathbf{r}) \times \partial_{z} \mathbf{m}(\mathbf{r})\right]_{i}\left[\mathbf{m}\left(\mathbf{r}^{\prime}\right) \times \partial_{z^{\prime}} \mathbf{m}\left(\mathbf{r}^{\prime}\right)\right]_{j} .
\end{aligned}
$$

(a)
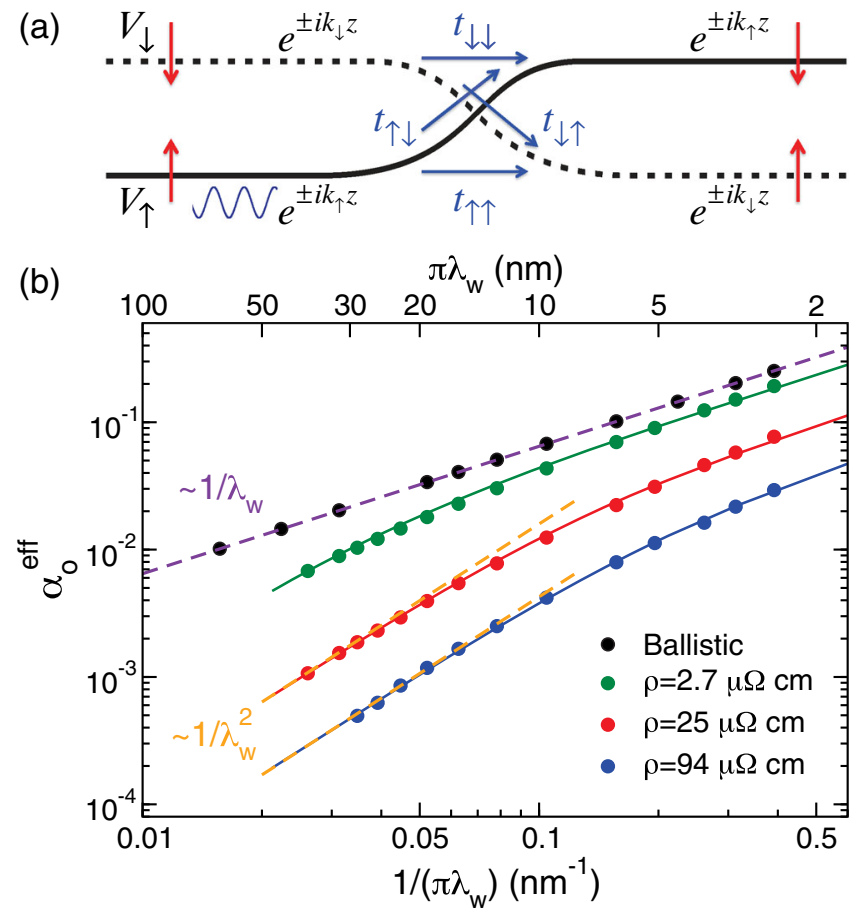

FIG. 3 (color online). (a) Illustration of electronic transport in a two-band, free-electron DW. The global quantization axis of the system is defined by the majority- and minority-spin states in the left domain. (b) Calculated $\alpha_{\mathrm{o}}^{\text {eff }}$ for two-band free-electron DWs as a function of $1 /\left(\pi \lambda_{w}\right)$ on a log-log scale. The black circles show the calculated results for the clean DWs, which are in perfect agreement with the analytical model Eq. (2), shown as a dashed violet line. When disorder (characterized by the resistivity $\rho$ calculated for the corresponding collinear magnetization) is introduced, $\alpha_{0}^{\text {eff }}$ shows a transition from a linear dependence on $1 / \lambda_{w}$ for narrow DWs to a quadratic behaviour for wide DWs. The solid lines are fits using Eq. (3). The dashed orange lines illustrate quadratic behavior.

Here $\alpha^{\prime}$ is a material parameter with dimensions of length squared and $D$ is a correlation function with an effective correlation length $l_{0}$. In practice, we use $D\left(\mathbf{r}, \mathbf{r}^{\prime} ; l_{0}\right)=$ $\left(1 / \sqrt{\pi} A l_{0}\right) e^{-\left(z-z^{\prime}\right)^{2} / l_{0}^{2}}$, which reduces to $\delta\left(\mathbf{r}-\mathbf{r}^{\prime}\right)$ in the diffusive limit $\left(l_{0} \ll \lambda_{w}\right)$ and reproduces earlier results [15-17]. In the ballistic limit, both $\alpha^{\prime}$ and $l_{0}$ are infinite, but the product $\alpha^{\prime} D\left(\mathbf{r}, \mathbf{r}^{\prime} ; l_{0}\right)=\alpha^{\prime} /\left(\sqrt{\pi} A l_{0}\right)$ is finite and related to the Sharvin conductance of the system [33], consistent with Eq. (2). We then fit the calculated values of $\alpha_{0}^{\text {eff }}$ shown in Fig. 3(b) using Eq. (3) [25]. With the parameters $\alpha^{\prime}$ and $l_{0}$ listed in Table $\mathrm{I}$, the fit is seen to be excellent over the whole range of $\lambda_{w}$. The out-of-plane damping enhancement arises from the pumped spin current $\mathbf{j}_{s}^{\prime} \sim \partial_{t} \mathbf{m} \times \partial_{z} \mathbf{m}$ in a magnetization texture [15,17], where the magnitude of $\mathbf{j}_{s}^{\prime}$ is related to the conductivity [15]. This is the reason why $\alpha^{\prime}$ is larger in a system with a lower resistivity in Table I. $l_{0}$ is a measure of how far the pumped transverse spin current can propagate before being absorbed by the local magnetization. It is worth distinguishing the relevant characteristic lengths in microscopic 
TABLE I. Fit parameters used to describe the damping shown in Fig. 1 for Permalloy DWs and in Fig. 3 for free-electron DWs with Eq. (3). The resistivity is determined for the corresponding collinear magnetization.

\begin{tabular}{lccc}
\hline \hline System & $\rho(\mu \Omega \mathrm{cm})$ & $\alpha^{\prime}\left(\mathrm{nm}^{2}\right)$ & $l_{0}(\mathrm{~nm})$ \\
\hline Free electron & 2.69 & 45.0 & 13.8 \\
Free electron & 24.8 & 1.96 & 4.50 \\
Free electron & 94.3 & 0.324 & 2.78 \\
\hline Py $\left(\xi_{\text {SO }}=0\right)$ & 0.504 & 23.1 & 28.3 \\
Py $\left(\xi_{\text {SO }} \neq 0\right)$ & 3.45 & 5.91 & 13.1 \\
\hline \hline
\end{tabular}

spin transport that define the diffusive regimes for different transport processes. The mean free path $l_{m}$ is the length scale for diffusive charge transport. The spin-flip diffusion length $l_{\text {sf }}$ characterizes the length scale for diffusive transport of a longitudinal spin current, and $l_{0}$ is the corresponding length scale for transverse spin currents. Only when the system size is larger than the corresponding characteristic length can transport be described in a local approximation.

We can use Eq. (3) to fit the calculated $\alpha_{0}^{\text {eff }}$ shown in Fig. 1 for Permalloy DWs. The results are shown in Fig. 4. Since the values of $\alpha_{0}^{\text {eff }}$ we calculate for Néel and Bloch DWs are nearly identical, we take their average for the SOC case. Intuitively, we would expect the out-of-plane damping for a highly disordered alloy like Permalloy to be in the diffusive regime corresponding to a short $l_{0}$. But the fitted values of $l_{0}$ are remarkably large, as long as $28.3 \mathrm{~nm}$ without SOC. With SOC, $l_{0}$ is reduced to $13.1 \mathrm{~nm}$ implying that nonlocal damping can play an important role in nanoscale magnetization textures in Permalloy, whose length scale in experiment is usually about $100 \mathrm{~nm}$ and can be reduced to be even smaller than $l_{0}$ by manipulating the shape anisotropy of experimental samples [34,35].

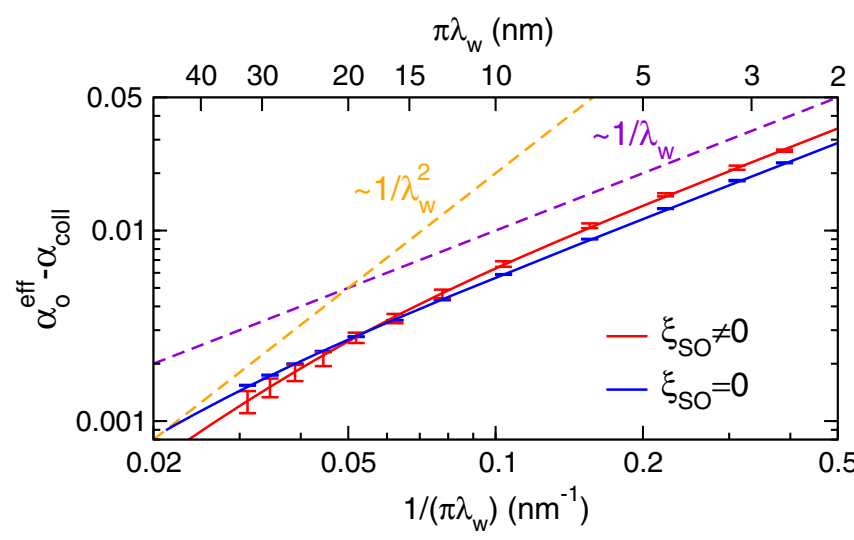

FIG. 4 (color online). Calculated out-of-plane damping $\alpha_{\mathrm{o}}^{\mathrm{eff}}-\alpha_{\text {coll }}$ from Fig. 1 plotted as a function of $1 /\left(\pi \lambda_{w}\right)$ on a $\log -\log$ scale. The solid lines are fitted using Eq. (3). The dashed violet and orange lines illustrate linear and quadratic behavior, respectively.
As shown in Table I, $l_{0}$ is positively correlated with the conductivity. The large value of $l_{0}$ and the low resistivity of Permalloy can be qualitatively understood in terms of its electronic structure and spin-dependent scattering. The $\mathrm{Ni}$ and Fe potentials seen by majority-spin electrons around the Fermi level in Permalloy are almost identical [25] so that they are only very weakly scattered. The $\mathrm{Ni}$ and Fe potentials seen by minority-spin electrons are however quite different leading to strong scattering in transport. The strongly asymmetric spin-dependent scattering can also be seen in the resistivity of Permalloy calculated without SOC, where $\rho_{\downarrow} / \rho_{\uparrow}>200[21,36]$. As a result, conduction in Permalloy is dominated by the weakly scattered majorityspin electrons resulting in a low total resistivity and a large value of $l_{0}$. This short-circuit effect is only slightly reduced by SOC-induced spin-flip scattering because the SOC in $3 d$ transition metals is in energy terms small compared to the bandwidth and exchange splitting. Indeed, $\alpha_{0}^{\text {eff }}-\alpha_{\text {coll }}$ calculated with SOC (the red curve in Fig. 4) shows a greater curvature at large widths than without SOC but is still quite different from the quadratic function characteristic of diffusive behavior for the widest DWs we could study.

Both $\alpha_{i}^{\text {eff }}$ and $\alpha_{0}^{\text {eff }}$ originate from locally pumped spin currents proportional to $\mathbf{m} \times \partial_{t} \mathbf{m}$. Because of the spatially varying magnetization, the spin currents pumped to the left and right do not cancel exactly and the net spin current contains two components, $\mathbf{j}_{s}^{\prime \prime} \sim-\mathbf{m} \times \partial_{z} \partial_{t} \mathbf{m}$ [19] and $\mathbf{j}_{s}^{\prime} \sim$ $\partial_{t} \mathbf{m} \times \partial_{z} \mathbf{m}[15,17]$. For out-of-plane damping, $\partial_{z} \mathbf{m}$ is perpendicular to $\partial_{t} \mathbf{m}$ so there is large enhancement due to the lowest order derivative. For the rigid motion of a 1D DW, $\partial_{z} \mathbf{m}$ is parallel to $\partial_{t} \mathbf{m}$ so that $\mathbf{j}_{s}^{\prime}$ vanishes. The enhancement of in-plane damping arising from $\mathbf{j}_{s}^{\prime \prime}$ due to the higher-order spatial derivative of magnetization is then smaller.

Conclusions.-We have discovered an anisotropic texture-enhanced Gilbert damping in Permalloy DWs using first-principles calculations. The findings are expressed in a form [Eqs. (1) and (3)] suitable for application to micromagnetic simulations of the dynamics of magnetization textures. The nonlocal character of the magnetization dissipation suggests that field- and/or current-driven DW motion, which is always assumed to be in the diffusive limit, needs to be reexamined. The more accurate form of the damping that we propose can be used to deduce the CITs in magnetization textures where the usual way to study them quantitatively is by comparing experimental observations with simulations.

Current-driven DWs move with velocities that are proportional to $\beta / \alpha$ where $\beta$ is the nonadiabatic spin transfer torque parameter. The order of magnitude spread in values of $\beta$ deduced for Permalloy from measurements of the velocities of vortex DWs [37-40] may be a result of assuming that $\alpha$ is a scalar constant. Our predictions can be tested by reexamining these studies using the expressions for $\alpha$ given in this paper as input to micromagnetic calculations. 
We would like to thank Geert Brocks and Taher Amlaki for useful discussions. This work was financially supported by the "Nederlandse Organisatie voor Wetenschappelijk Onderzoek" (NWO) through the research program of "Stichting voor Fundamenteel Onderzoek der Materie" (FOM) and the supercomputer facilities of NWO "Exacte Wetenschappen (Physical Sciences)." It was also partly supported by the Royal Netherlands Academy of Arts and Sciences (KNAW). A. B. acknowledges the Research Council of Norway, Grant No. 216700.

*Present address: Institut für Physik, Johannes GutenbergUniversität Mainz, Staudingerweg 7, 55128 Mainz, Germany. zyuan@uni-mainz.de

[1] S. M. Mohseni, S. R. Sani, J. Persson, T. N. A. Nguyen, S. Chung, Y. Pogoryelov, P. K. Muduli, E. Iacocca, A. Eklund, R. K. Dumas, S. Bonetti, A. Deac, M. A. Hoefer, and J. Åkerman, Science 339, 1295 (2013).

[2] X. Z. Yu, Y. Onose, N. Kanazawa, J. H. Park, J. H. Han, Y. Matsui, N. Nagaosa, and Y. Tokura, Nature (London) 465, 901 (2010).

[3] A. Fert, V. Cros, and J. Sampaio, Nat. Nanotechnol. 8, 152 (2013).

[4] L. Thomas, R. Moriya, C. Rettner, and S. S. P. Parkin, Science 330, 1810 (2010).

[5] J. H. Franken, H. J. M. Swagten, and B. Koopmans, Nat. Nanotechnol. 7, 499 (2012).

[6] A. Brataas, A. D. Kent, and H. Ohno, Nat. Mater. 11, 372 (2012).

[7] J. C. Slonczewski, J. Magn. Magn. Mater. 159, L1 (1996).

[8] L. Berger, Phys. Rev. B 54, 9353 (1996).

[9] I. M. Miron, G. Gaudin, S. Auffret, B. Rodmacq, A. Schuhl, S. Pizzini, J. Vogel, and P. Gambardella, Nat. Mater. 9, 230 (2010).

[10] S. Emori, U. Bauer, S.-M. Ahn, E. Martinez, and G. S. D. Beach, Nat. Mater. 12, 611 (2013).

[11] O. Boulle, S. Rohart, L. D. Buda-Prejbeanu, E. Jué, I. M. Miron, S. Pizzini, J. Vogel, G. Gaudin, and A. Thiaville, Phys. Rev. Lett. 111, 217203 (2013).

[12] L. Liu, C.-F. Pai, Y. Li, H. W. Tseng, D. C. Ralph, and R. A. Buhrman, Science 336, 555 (2012).

[13] K.-S. Ryu, L. Thomas, S.-H. Yang, and S. Parkin, Nat. Nanotechnol. 8, 527 (2013).

[14] H. T. Nembach, J. M. Shaw, C. T. Boone, and T. J. Silva, Phys. Rev. Lett. 110, 117201 (2013).

[15] J. Foros, A. Brataas, Y. Tserkovnyak, and G. E. W. Bauer, Phys. Rev. B 78, 140402 (2008).

[16] Y. Tserkovnyak and C. H. Wong, Phys. Rev. B 79, 014402 (2009).

[17] S. Zhang and S. S.-L. Zhang, Phys. Rev. Lett. 102, 086601 (2009).

[18] E. M. Hankiewicz, G. Vignale, and Y. Tserkovnyak, Phys. Rev. B 78, 020404(R) (2008).
[19] Y. Tserkovnyak, E. M. Hankiewicz, and G. Vignale, Phys. Rev. B 79, 094415 (2009).

[20] Y. Li and W. E. Bailey, arXiv:1401.6467.

[21] A. A. Starikov, P. J. Kelly, A. Brataas, Y. Tserkovnyak, and G. E. W. Bauer, Phys. Rev. Lett. 105, 236601 (2010).

[22] K. M. D. Hals and A. Brataas, Phys. Rev. B 89, 064426 (2014).

[23] The definitions, conventions and methodology are the same as used by Z. Yuan, Y. Liu, A. A. Starikov, P. J. Kelly, and A. Brataas, Phys. Rev. Lett. 109, 267201 (2012).

[24] A. Brataas, Y. Tserkovnyak, and G. E. W. Bauer, Phys. Rev. Lett. 101, 037207 (2008); Phys. Rev. B 84, 054416 (2011); K. M. D. Hals, A. K. Nguyen, and A. Brataas, Phys. Rev. Lett. 102, 256601 (2009).

[25] See Supplemental Material at http://link.aps.org/ supplemental/10.1103/PhysRevLett.113.266603, which includes Refs. [26-30], for computational details; extracting $\alpha^{\prime \prime}$; the free-electron model using muffin-tin orbitals; fitting $\alpha^{\prime}$ and $l_{0}$; and band structures of $\mathrm{Ni}$ and $\mathrm{Fe}$ in Permalloy.

[26] K. Xia, M. Zwierzycki, M. Talanana, P. J. Kelly, and G. E. W. Bauer, Phys. Rev. B 73, 064420 (2006).

[27] O. K. Andersen, Z. Pawlowska, and O. Jepsen, Phys. Rev. B 34, 5253 (1986).

[28] I. Turek, V. Drchal, J. Kudrnovský, M. Šob, and P. Weinberger, Electronic Structure of Disordered Alloys, Surfaces and Interfaces (Kluwer, Boston-London-Dordrecht, 1997).

[29] P. Soven, Phys. Rev. 156, 809 (1967).

[30] S. Wang, Y. Xu, and K. Xia, Phys. Rev. B 77, 184430 (2008).

[31] R. A. Duine, Phys. Rev. B 79, 014407 (2009).

[32] A. K. Nguyen and A. Brataas, Phys. Rev. Lett. 101, 016801 (2008).

[33] It is analogous to the relation between the Drude conductivity $\sigma$ and the mean free path $l_{m}, \sigma=\left(2 e^{2} / h\right)\left(k_{F}^{2} / 3 \pi\right) l_{m}$. In ballistic systems, we have $\sigma \rightarrow \infty$ and $l_{m} \rightarrow \infty$, $\sigma / l_{m}=\left(2 e^{2} / h\right)\left(k_{F}^{2} / 3 \pi\right)=\left(4 G_{\mathrm{Sh}} / 3 A\right)$. Here we have used the Sharvin conductance of free electrons $G_{\mathrm{Sh}}=\left(2 e^{2} / h\right)$ $\left(A k_{F}^{2} / 4 \pi\right)$.

[34] O. Boulle, G. Malinowski, and M. Kläui, Mat. Science Eng. R 72, 159 (2011).

[35] A. Ben Hamida, O. Rousseau, S. Petit-Watelot, and M. Viret, Europhys. Lett. 94, 27002 (2011).

[36] J. Banhart, H. Ebert, and A. Vernes, Phys. Rev. B 56, 10165 (1997).

[37] M. Hayashi, L. Thomas, C. Rettner, R. Moriya, and S. S. P. Parkin, Appl. Phys. Lett. 92, 162503 (2008).

[38] S. Lepadatu, A. Vanhaverbeke, D. Atkinson, R. Allenspach, and C. H. Marrows, Phys. Rev. Lett. 102, 127203 (2009).

[39] M. Eltschka, M. Wötzel, J. Rhensius, S. Krzyk, U. Nowak, M. Kläui, T. Kasama, R. E. Dunin-Borkowski, L. J. Heyderman, H. J. van Driel, and R. A. Duine, Phys. Rev. Lett. 105, 056601 (2010).

[40] J.-Y. Chauleau, H. G. Bauer, H. S. Körner, J. Stigloher, M. Härtinger, G. Woltersdorf, and C. H. Back, Phys. Rev. B 89, 020403(R) (2014). 